\title{
RESEARCH
}

Open Access

\section{Quality of opioid prescribing in older adults with or without Alzheimer disease and related dementia}

Yu-Jung Jenny Wei ${ }^{1,2^{*}} \mathbb{D}$, Siegfried Schmidt ${ }^{3}$, Cheng Chen ${ }^{1}$, Roger B. Fillingim ${ }^{4,5}$, M. Carrington Reid ${ }^{6}$, Steven DeKosky ${ }^{7}$, Laurence Solberg ${ }^{8,9}$, Marco Pahor ${ }^{10}$, Babette Brumback ${ }^{11}$ and Almut G. Winterstein ${ }^{1,2,12}$

\begin{abstract}
Background: Pain is common among individuals with Alzheimer's disease and related dementias (ADRD), and use of opioids has been increasing over the last decade. Yet, it is unclear to what extent opioids are appropriately prescribed for patients with ADRD and whether the appropriateness of opioid prescribing differs by ADRD status. The objective of this study is to compare the quality of opioid prescribing among patients with or without ADRD who have chronic noncancer pain.

Methods: A nationally representative cohort study of Medicare beneficiaries aged 50 years or older who had chronic pain but who had no cancer, hospice, or palliative care from 2011 to 2015. Four indicators of potentially inappropriate opioid prescribing were measured in patients residing in communities (75,258 patients with and 435, 870 patients without ADRD); five indicators were assessed in patients in nursing homes (NHs) (37,117 patients with and 5128 patients without ADRD). Each indicator was calculated as the proportion of eligible patients with inappropriate opioid prescribing in the year after a chronic pain diagnosis. Differences in proportions between ADRD and non-ADRD groups were estimated using a generalized linear model adjusting for covariates through inverse probability weighting.

Results: Patients with ADRD versus those without had higher concurrent use of opioids and central nervous system-active drugs (community $44.1 \%$ vs $33.3 \%$; NH $58.8 \%$ vs $54.1 \%$, both $P<0.001$ ) and no opioids or scheduled pain medications for moderate or severe pain ( $\mathrm{NH} 60.1 \%$ vs $52.5 \%, P<0.001$ ). The ADRD versus non-ADRD group had higher use of long-term opioids for treating neuropathic pain in communities $(21.7 \%$ vs $19.5 \%, P=0.003)$ but lower use in $\mathrm{NHs}(26.9 \%$ vs $36.0 \%, P<0.001)$. Use of strong or high-dose opioids when naive to opioids (community $1.5 \%$ vs $2.8 \% ; \mathrm{NH} 2.5 \%$ vs $3.5 \%$ ) and use of contraindicated opioids (community $0.08 \%$ vs $0.12 \% ; \mathrm{NH} 0.05 \%$ vs $0.21 \%$ ) were rare for either group.

(Continued on next page)
\end{abstract}

\footnotetext{
* Correspondence: jenny.wei@cop.ufl.edu

'Department of Pharmaceutical Outcomes and Policy, University of Florida

College of Pharmacy, 1225 Center Drive, Health Professions Nursing

Pharmacy Building, Room 3321, Gainesville, FL 32610, USA

${ }^{2}$ Center for Drug Evaluation and Safety, University of Florida, Gainesville, FL, USA

Full list of author information is available at the end of the article
}

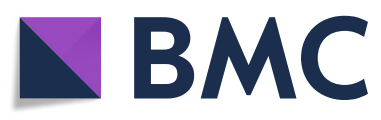

(c) The Author(s). 2021 Open Access This article is licensed under a Creative Commons Attribution 4.0 International License, which permits use, sharing, adaptation, distribution and reproduction in any medium or format, as long as you give appropriate credit to the original author(s) and the source, provide a link to the Creative Commons licence, and indicate if changes were made. The images or other third party material in this article are included in the article's Creative Commons licence, unless indicated otherwise in a credit line to the material. If material is not included in the article's Creative Commons licence and your intended use is not permitted by statutory regulation or exceeds the permitted use, you will need to obtain permission directly from the copyright holder. To view a copy of this licence, visit http://creativecommons.org/licenses/by/4.0/ The Creative Commons Public Domain Dedication waiver (http://creativecommons.org/publicdomain/zero/1.0/) applies to the data made available in this article, unless otherwise stated in a credit line to the data. 
(Continued from previous page)

Conclusion: Potential inappropriate opioid prescribing in 2 areas of pain care was more common among patients with ADRD than among patients without ADRD in community or NH settings. Further studies aimed at understanding the factors and effects associated with opioid prescribing patterns that deviate from guidelines are warranted.

Keywords: Alzheimer's disease and related dementias, Prescription opioids, Inappropriate prescribing

\section{Introduction}

Pain is common among older adults with Alzheimer disease and related dementias (ADRD), with up to $60 \%$ of affected patients in communities and $80 \%$ of those in nursing homes (NHs) reporting regular pain [1]. Uncontrolled pain in ADRD contributes to poor physical function and mental disorders, notably depression, behavioral, and other psychiatric symptoms [2, 3].

Pharmacological treatment is commonly used to manage older adults' pain [4]. Historical data have shown lower use of analgesics in patients with ADRD vs those without [5]. Memory decline and decreased communication skills among patients with cognitive impairment have been implicated in the underuse of analgesics $[5,6]$. Findings of recent population-based studies, however, suggest that patients with ADRD were equally or more likely than patients without ADRD to receive analgesics for pain management [7-10]. The increasing analgesic use in patients with ADRD may result from increasing clinical awareness of the need for improved pain assessment and management in this patient population $[7,10]$.

Inappropriate medication prescribing practice is one of the primary reasons for uncontrolled pain $[8,10-12]$. Opioids are commonly used to treat pain in older adults, with nearly 1 in 5 older adults filling at least 1 prescription opioid in 2015 [13]. To date, it remains unclear the extent to which opioids are being prescribed appropriately for older adults with chronic noncancer pain and whether the quality of opioid prescribing differs between those with or without ADRD. To address this question, we assessed and compared quality indicators of appropriate opioid prescribing practices commonly described in guidelines and published literature for the management of chronic pain among older adults with ADRD vs those without (Table 1). The selected quality indicators were assessed in both community and $\mathrm{NH}$ settings.

\section{Methods}

\section{Study design and source}

We conducted a cohort study of a $5 \%$ random sample of Medicare beneficiaries linked to the Minimum Data Set, version 3.0 (MDS 3.0) from 2011 to 2015. Medicare data contain fee-for-service enrollees' medical billing records for Parts A, B, and D (prescription drugs) and beneficiary-level sociodemographic characteristics, enrollment status, and presence of 27 chronic conditions, including ADRD [14]. The University of Florida Institutional Review Board approved and waived patient informed consent for this study.

The latest version of a federally mandated clinical assessment, MDS 3.0, collects data from all residents of Medicare- or Medicaid-certified NHs [15]. Most relevant to quality measures of opioid prescribing is MDS 3.0 Section J Health Condition, which documents selfreported pain intensity using numeric rating or verbal descriptor scale, supplemented with nursing staffassessed pain using the checklist of nonverbal pain indicators for nonverbal residents, as well as the use of scheduled pain medication regimens. We used these pain-related data in MDS 3.0 to assess the appropriateness of opioid prescribing for moderate to severe pain among patients in NHs (Table 1).

\section{Study sample}

The study sample included adults 50 years of age or older who had (1) at least 1 primary or secondary diagnosis of a chronic pain condition; (2) no diagnosis or procedures indicating cancer, hospice, or palliative care services; and (3) continuous enrollment in Medicare Parts A, B, and D for at least 18 months between 2011 and 2015. The 18-month period included a 6-month baseline before (for determining demographic and clinical characteristics, pain types, and history of opioid use) and a 12-month follow-up after a chronic pain diagnosis (for assessing the appropriateness of opioid prescribing). For each patient, we randomly selected one 18-month continuous enrollment period as an observation unit. We excluded patients with a health-maintenanceorganization or an employer-sponsored insurance plan (owing to lack of complete encounters from in- and outpatient settings) during the 18-month period. The diagnostic and procedure codes for conditions and services considered in the sample selection are given in Supplement eTable 1.

We further categorized eligible patients based on residential status (community vs $\mathrm{NH}$ ) because of the differences in patient characteristics and ADRD severity associated with this factor $[16,17]$. Patients were classified as $\mathrm{NH}$ residents if they had at least 1 episode of a long $\mathrm{NH}$ stay ( $>100$ days, measured based on MDS 3.0 
Table 1 Quality measures of potentially inappropriate opioid prescribing among older adults ( $\geq 50$ years) with chronic noncancer pain

\begin{tabular}{|c|c|c|c|c|}
\hline \multirow{2}{*}{$\begin{array}{l}\text { Measure } \\
\text { domain }\end{array}$} & \multirow{2}{*}{$\begin{array}{l}\text { Indicators of inappropriate opioid } \\
\text { prescribing }\end{array}$} & \multicolumn{3}{|c|}{ Operationalization with 2011-2015 Medicare and MDS 3.0 data } \\
\hline & & Denominator & Numerator & Exclusion \\
\hline $\begin{array}{l}\text { 1. Opioids } \\
\text { contraindicated } \\
\text { for older adults }\end{array}$ & $\begin{array}{l}\text { - Use of meperidine, propoxyphene, } \\
\text { pentazocine, butorphanol, and } \\
\text { nalbuphine }\end{array}$ & Older adults with chronic pain & Patients with contraindicated opioids & $\begin{array}{l}\text { Cancer, } \\
\text { hospice, } \\
\text { or }\end{array}$ \\
\hline \multirow[t]{3}{*}{$\begin{array}{l}\text { 2. Opioid-naïve } \\
\text { patients }\end{array}$} & $\begin{array}{l}\text { - Use of long-acting (LA) or extended- } \\
\text { release (ER) prescription opioids for } \\
\text { opioid-naïve patients }\end{array}$ & $\begin{array}{l}\text { Patients with chronic pain who } \\
\text { were naïve to opioids (i.e., no } \\
\text { opioids in } 6 \text { months before an index } \\
\text { chronic pain diagnosis [baseline]) }\end{array}$ & $\begin{array}{l}\text { Patients with LA/ER prescription opioids } \\
\text { or using medications without evidence } \\
\text { of drug tolerance (i.e., receive } \geq 60 \text { daily } \\
\text { MME) for a week or longer }\end{array}$ & $\begin{array}{l}\text { palliative } \\
\text { care }\end{array}$ \\
\hline & $\begin{array}{l}\text { - Use of high-dose prescription opi- } \\
\text { oids for opioid-naïve patients }\end{array}$ & & Patients with a daily dose of $\geq 90 \mathrm{MME}$ & \\
\hline & - Composite of any & & $\begin{array}{l}\text { Patients with } L A / E R \text { prescription opioids } \\
\text { or with a daily dose of } \geq 90 \mathrm{MME}\end{array}$ & \\
\hline \multirow{2}{*}{$\begin{array}{l}\text { 3. Patients with } \\
\text { neuropathic } \\
\text { pain }\end{array}$} & • Long-term (> 90 days) use of opioids & $\begin{array}{l}\text { Older adults with only neuropathic } \\
\text { pain as the index diagnosis }\end{array}$ & Patients with long-term use of opioids & \\
\hline & & $\begin{array}{l}\text { Sensitivity analysis: older adults with } \\
\text { only neuropathic pain who had no } \\
\text { musculoskeletal or idiopathic pain } \\
\text { during the 6-month baseline }\end{array}$ & & \\
\hline $\begin{array}{l}\text { 4. Concurrent } \\
\text { use of opioids } \\
\text { and CNS } \\
\text { depressants }\end{array}$ & $\begin{array}{l}\text { - Concurrent use of opioids with any } \\
\text { CNS drugs that Beers Criteria' } \\
\text { recommends against }\end{array}$ & $\begin{array}{l}\text { Older adults with chronic pain and } \\
\text { using prescription opioids }\end{array}$ & $\begin{array}{l}\text { Patients with concurrent use of Beers } \\
\text { Criteria }^{1} \text { CNS depressants for } \geq 7 \text { days }\end{array}$ & \\
\hline \multirow{3}{*}{$\begin{array}{l}\text { 5. Patients with } \\
\text { moderate to } \\
\text { severe pain } \\
\text { (NHs only) }\end{array}$} & $\begin{array}{l}\text { - No prescription opioids within } 30 \\
\text { days before or after reporting } \\
\text { moderate to severe pain }\end{array}$ & $\begin{array}{l}\text { Older adults with moderate to } \\
\text { severe chronic pain }\end{array}$ & $\begin{array}{l}\text { Patients with no prescription opioids } \\
\text { within } 30 \text { days before or after the } \\
\text { qualifying pain score }\end{array}$ & \\
\hline & $\begin{array}{l}\text { - No use of scheduled pain } \\
\text { medication regimen in the } 5 \text { days } \\
\text { before reporting moderate to severe } \\
\text { chronic pain (defined based on MDS } \\
3.0 \text { pain assessment) }\end{array}$ & & $\begin{array}{l}\text { Patients with no scheduled pain } \\
\text { medication regimen (defined based on } \\
\text { MDS } 3.0 \text { item J0100A) }\end{array}$ & \\
\hline & - Composite of any & & $\begin{array}{l}\text { Patients with no prescription opioids or } \\
\text { no scheduled pain medications }\end{array}$ & \\
\hline
\end{tabular}

Abbreviations: CNS central nervous system, MDS 3.0 Minimum Data Set, version 3.0, MME morphine milligram equivalent, $N H s$ nursing homes

${ }^{1}$ American Geriatrics Society 2015 updated Beers criteria for potentially inappropriate medication use in older adults

assessment dates) during the 12-month follow-up period [18]. The choice of using $>100$ days to define long-stay $\mathrm{NH}$ residents is consistent with the definition set by the US Center for Medicare and Medicaid Services (CMS) [18]. Patients were classified as community dwellers if they had no or a short $\mathrm{NH}$ stay ( $\leq 100$ days). We then created 4 community-dwelling cohorts and $5 \mathrm{NH}$ cohorts, with each cohort corresponding to patients who were eligible for the denominator of a specific quality indicator (Supplement eFigure 1).

\section{Prescription opioid and other pain medications}

We captured prescription opioids and other pain medications (including non-opioids analgesics and adjuvant treatments) using Medicare Part D files and measured drug use in the year after a chronic pain diagnosis (Supplement eTable 2). For opioids, we excluded injectable opioids used primarily in inpatient, rectal dosage forms that are rarely used, and buprenorphine in sublingual form and combined buprenorphine-naloxone products, which are used for addiction treatment. We converted the dose of each filled opioid prescription to daily morphine milligram equivalents (MMEs) by multiplying the quantity of opioids prescribed per day by the strength and MME conversion factor [19].

\section{Quality indicators of inappropriate opioid prescribing}

Quality indicators of inappropriate opioid prescribing were defined based on core principles of clinical guidelines and published literature for the management of chronic noncancer pain in older adults (Supplement eTable 3). Four major quality indicators were selected and measured in the year after a chronic pain diagnosis: (1) use strong or high-dose (defined as $\geq 90$ MME per day [20]) opioid regimen among opioid-naïve patients who had no prescription opioid use at baseline; (2) longterm use ( $\geq 90$ days $[21,22]$ ) of opioids for patients with neuropathic pain as the index diagnosis; (3) concurrent use of opioids with other central nervous system (CNS) depressant drugs for 7 or more days [23, 24]; and (4) use 
of opioids contraindicated for older adults [24-26]. An additional (fifth) indicator was measured only in $\mathrm{NH}$ patients based on MDS 3.0-the absence of opioid prescription or the absence of a scheduled pain treatment regimen within days of reporting moderate to severe pain [26, 27]. A detailed definition of each indicator is given in Table 1. Each indicator was calculated as the proportion of eligible patients with inappropriate opioid prescribing following the year after a chronic pain diagnosis.

\section{Statistical analysis}

In both community and NH settings, we compared quality indicators between patients with versus without ADRD. Patients with ADRD were identified based on the Chronic Condition Data Warehouse flags as having at least one institutional (including inpatient, outpatient, skilled nursing facility, home healthcare, and hospice care) or office-based medical claims with any of 24 diagnostic codes for ADRD [14]. Because the ADRD and non-ADRD groups differed in many characteristics that may be associated with opioid prescribing practices, we used an inverse probability weighting (IPW) approach to balance differences in group characteristics that may bias outcome estimates, while retaining all study patients in the analysis [28]. In IPW, data from each patient were weighted by the inverse of the estimated probability of ADRD status conditional on measured baseline characteristics through a logistic regression model. Separate models were created to generate IPW weights using the characteristics of patient cohorts in communities (Table 2) and in NHs (Table 3). In the NH sample, in addition to characteristics measured from Medicare claims data, we also adjusted for three characteristics extracted from MDS 3.0 data-(1) Patient Health Questionnaire (PHQ)-9 depression symptoms (range 0-27), classified as no (0-4), mild (5-9), moderate (10-14), and severe depression ( $\geq 15)$ [29]; (2) activities of daily living (ADLs, range 0-28), classified into no (0-7), mild (8$14)$, moderate (15-21), and severe $(\geq 21)$ dependence [30]; and (3) body mass index (BMI), calculated based on MDS-3.0 documented weight and height and classified into underweight (BMI $\left.<18.5 \mathrm{~kg} / \mathrm{m}^{2}\right)$, normal (18.524.9), obsess (25.0-29.9), and overweight $(\geq 30)$ [31]. We refrained from adjusting for MDS 3.0-assessed cognitive function and aggressive behaviors in the $\mathrm{NH}$ sample due to only a small proportion ( $<5 \%$, data not shown) of non-ADRD residents having moderate to severe cognitive function or aggressive behaviors. Adjustment of these two variables along with other characteristics would have produced extreme IPW weights, an indicative of violation of positivity assumption (i.e., probability of any patient having exposure [i.e., ADRD vs non-
ADRD] is positive, nonzero with each stratum of covariate combination) [32].

We reported the weighted proportion of patients with chronic pain who received any pain medications, overall and by therapeutic classes (opioids, non-opioids, and adjuvants by therapeutic class) during the 12-month follow-up in ADRD or non-ADRD groups, by residential status. For each quality indicator and each setting, we also reported the weighted proportion of patients with inappropriate opioid prescribing between ADRD and non-ADRD groups. We estimated the proportion differences between groups and their 95\% CIs using generalized linear models. All analyses were performed from April 2019 to April 2020 using SAS, version 9.4 (SAS Institute Inc). Statistical significance was set at $P<0.05$, and all tests were 2 -sided.

\section{Results}

Of 553,373 Medicare beneficiaries with chronic pain identified, 75,258 patients with ADRD and 435,870 patients without ADRD were living in communities (Table 2), and 37,117 patients with ADRD and 5128 patients without ADRD were residing in NHs (Table 3). In both settings, compared with patients without ADRD, those with ADRD were older (mean [SD] age: in community, 79 [10] vs 71 [9] years; in $\mathrm{NH}, 82$ [10] vs 73 [13] years; both $P<.001)$ and were more likely to be female (community, $71 \%$ vs $63 \%$; $\mathrm{NH}, 75 \%$ vs $64 \%$; both $P<.001)$. The proportion of nonwhite was higher in patients with vs without ADRD in communities $(21 \%$ vs $18 \%, P<.001)$ but was similar in NHs $(20 \%$ vs $19 \% ; P=$ .205). After IPW, distributions of all measured baseline characteristics were well balanced between ADRD and non-ADRD groups in all patient cohorts in communities and NHs (Supplement eTables 4 and 5), with standardized mean differences for characteristics less than 0.1 (Supplement eFigures 2 and 3).

\section{Receipt of pain medications in patients with or without ADRD}

After IPW, patients with ADRD were less likely than their non-ADRD counterparts to receive any prescription pain medication in the year after a chronic pain diagnosis in communities $(66.6 \%$ vs $67.6 \%$; difference, $1.0 \%$ [95\% CI, -1.4 to $-0.7 \%$; $P<.001)$ and $\mathrm{NHs}(64.5 \%$ vs $74.9 \%$; difference, $-10.4 \%$ [95\% CI, -11.7 to $-9.1 \%$; $P<.001$ ) (Table 4 ). In both settings, analyses by therapeutic classes of analgesics showed lower use of opioids (community, $45.1 \%$ vs $48.1 \%$; difference, $-3.0 \%$ [95\% CI, -3.4 to $-2.6 \%$ ]; $\mathrm{NH}, 47.9 \%$ vs $60.7 \%$; difference, $-12.8 \%$ [95\% CI, -14.2 to $-11.3 \%$ ]; both $P<.001$ ) or lower use of non-opioids (community, $30.3 \%$ vs $32.7 \%$; difference, $-2.4 \%$ [ $95 \% \mathrm{CI},-2.8$ to $-2.1 \%$; $\mathrm{NH}, 18.0 \%$ vs $21.9 \%$; difference, $-3.9 \%[95 \% \mathrm{CI},-5.1$ to $-2.7 \%]$; both 
Table 2 Demographic and clinical characteristics of community-dwelling older patients with chronic pain with or without ADRD stratified by cohort

\begin{tabular}{|c|c|c|c|c|c|c|c|c|c|c|c|c|}
\hline \multirow[t]{2}{*}{$\begin{array}{l}\text { Baseline } \\
\text { characteristic }^{a}\end{array}$} & \multicolumn{2}{|c|}{$\begin{array}{l}\text { Patients with } \\
\text { chronic pain, \% }\end{array}$} & \multirow{3}{*}{$\begin{array}{l}P- \\
\text { value }^{\mathrm{d}}\end{array}$} & \multicolumn{2}{|c|}{$\begin{array}{l}\text { Opioid-naive } \\
\text { patients, } \%\end{array}$} & \multirow{3}{*}{$\begin{array}{l}P \text { - } \\
\text { value }^{\mathrm{d}}\end{array}$} & \multicolumn{2}{|c|}{$\begin{array}{l}\text { Patients with } \\
\text { neuropathic pain, } \\
\%\end{array}$} & \multirow{3}{*}{$\begin{array}{l}P \text { - } \\
\text { value }^{d}\end{array}$} & \multicolumn{2}{|c|}{$\begin{array}{l}\text { Patients with } \\
\text { opioid } \\
\text { prescription, \% }\end{array}$} & \multirow{3}{*}{$\begin{array}{l}P \text { - } \\
\text { value }\end{array}$} \\
\hline & $\begin{array}{l}\text { With } \\
\text { ADRD }\end{array}$ & $\begin{array}{l}\text { Without } \\
\text { ADRD }\end{array}$ & & $\begin{array}{l}\text { With } \\
\text { ADRD }\end{array}$ & $\begin{array}{l}\text { Without } \\
\text { ADRD }\end{array}$ & & $\begin{array}{l}\text { With } \\
\text { ADRD }\end{array}$ & $\begin{array}{l}\text { Without } \\
\text { ADRD }\end{array}$ & & $\begin{array}{l}\text { With } \\
\text { ADRD }\end{array}$ & $\begin{array}{l}\text { Without } \\
\text { ADRD }\end{array}$ & \\
\hline Total no. & 75,258 & 435,870 & & 48,182 & 304,009 & & 9775 & 64,692 & & 27,801 & 197,886 & \\
\hline \multicolumn{13}{|l|}{ Age, $y$} \\
\hline Mean (SD) & $\begin{array}{l}79.3 \\
(10.4)\end{array}$ & $70.9(9.4)$ & $<.001$ & $\begin{array}{l}80.4 \\
(10.1)\end{array}$ & 72.1 (8.8) & $<.001$ & $\begin{array}{l}76.5 \\
(10.1)\end{array}$ & $69.7(9.3)$ & $<.001$ & $\begin{array}{l}77.8 \\
(10.6)\end{array}$ & $69.2(9.8)$ & $<.001$ \\
\hline $50-64$ & 9.4 & 19.3 & & 7.5 & 13.1 & & 12.5 & 23.2 & & 11.9 & 27.2 & \\
\hline $65-74$ & 19.3 & 48.0 & & 17.1 & 50.9 & & 26.2 & 48.0 & & 22.5 & 44.6 & \\
\hline $75-84$ & 36.3 & 24.7 & & 36.4 & 27.0 & & 38.1 & 22.6 & & 36.1 & 21.7 & \\
\hline$\geq 85$ & 35.0 & 8.0 & & 38.9 & 8.9 & & 23.1 & 6.1 & & 29.5 & 6.5 & \\
\hline Female & 71.1 & 63.1 & $<.001$ & 69.5 & 62.4 & $<.001$ & 67.3 & 62.0 & $<.001$ & 73.5 & 64.0 & $<.001$ \\
\hline \multicolumn{13}{|l|}{ Race/ethnicity } \\
\hline White & 78.7 & 82.0 & $<.001$ & 78.0 & 82.6 & $<.001$ & 76.8 & 82.1 & $<.001$ & 79.4 & 81.2 & $<.001$ \\
\hline Black & 11.7 & 10.4 & & 11.3 & 9.1 & & 12.4 & 10.3 & & 12.3 & 12.3 & \\
\hline Others $^{b}$ & 9.6 & 7.6 & & 10.6 & 8.3 & & 10.8 & 7.6 & & 8.2 & 6.4 & \\
\hline \multicolumn{13}{|l|}{ US region } \\
\hline Northeast & 21.0 & 18.9 & $<.001$ & 24.7 & 20.9 & $<.001$ & 20.7 & 19.6 & $<.001$ & 15.1 & 15.0 & $<.001$ \\
\hline Midwest & 21.7 & 24.5 & & 21.5 & 24.7 & & 19.9 & 23.9 & & 22.0 & 24.3 & \\
\hline South & 40.1 & 38.4 & & 36.8 & 36.1 & & 42.3 & 39.0 & & 45.6 & 42.8 & \\
\hline West or other regions & 17.2 & 18.2 & & 17.1 & 18.3 & & 17.1 & 17.5 & & 17.2 & 17.8 & \\
\hline Low-income subsidy & 44.0 & 34.5 & $<.001$ & 41.2 & 28.5 & $<.001$ & 46.6 & 35.7 & $<.001$ & 47.9 & 42.3 & $<.001$ \\
\hline $\begin{array}{l}\text { Tobacco or alcohol } \\
\text { use }\end{array}$ & 6.7 & 6.5 & .057 & 4.6 & 4.1 & $<.001$ & 7.3 & 6.7 & .021 & 9.3 & 9.5 & .391 \\
\hline Drug use disorder & 2.3 & 1.6 & $<.001$ & 1.1 & 0.6 & $<.001$ & 3.0 & 1.8 & $<.001$ & 3.9 & 2.7 & $<.001$ \\
\hline \multicolumn{13}{|l|}{ Index pain diagnosis ${ }^{c}$} \\
\hline Musculoskeletal & 88.8 & 86.7 & $<.001$ & 89.4 & 87.4 & $<.001$ & 24.5 & 19.7 & $<.001$ & 88.0 & 86.3 & $<.001$ \\
\hline Neuropathic & 13.0 & 14.8 & $<.001$ & 12.2 & 14.3 & $<.001$ & 100.0 & 100.0 & NA & 14.2 & 15.2 & $<.001$ \\
\hline Idiopathic & 2.4 & 2.0 & $<.001$ & 1.2 & 0.8 & $<.001$ & 1.2 & 0.7 & $<.001$ & 4.1 & 3.6 & $<.001$ \\
\hline \multicolumn{13}{|c|}{ Comorbidity affecting pain treatment } \\
\hline Cardiovascular disease & 85.4 & 68.1 & $<.001$ & 83.3 & 65.0 & $<.001$ & 86.8 & 70.3 & $<.001$ & 87.5 & 71.2 & $<.001$ \\
\hline Pulmonary condition & 52.9 & 40.7 & $<.001$ & 47.9 & 35.9 & $<.001$ & 53.5 & 41.0 & $<.001$ & 59.2 & 46.5 & $<.001$ \\
\hline Diabetes & 39.8 & 34.3 & $<.001$ & 38.0 & 32.4 & $<.001$ & 59.4 & 47.7 & $<.001$ & 42.6 & 36.7 & $<.001$ \\
\hline Mental disorder & 37.9 & 17.6 & $<.001$ & 32.9 & 13.2 & $<.001$ & 37.5 & 18.8 & $<.001$ & 43.5 & 22.7 & $<.001$ \\
\hline $\begin{array}{l}\text { Gastrointestinal tract } \\
\text { disorder }\end{array}$ & 27.2 & 13.7 & $<.001$ & 23.8 & 11.4 & $<.001$ & 26.8 & 14.2 & $<.001$ & 30.6 & 15.9 & $<.001$ \\
\hline Urinary tract infection & 24.6 & 9.4 & $<.001$ & 22.1 & 8.1 & $<.001$ & 22.3 & 10.0 & $<.001$ & 26.9 & 10.7 & $<.001$ \\
\hline Kidney disease & 21.8 & 11.2 & $<.001$ & 19.3 & 9.6 & $<.001$ & 24.3 & 13.1 & $<.001$ & 24.7 & 13.0 & $<.001$ \\
\hline Fall or fracture & 19.5 & 5.5 & $<.001$ & 14.7 & 3.2 & $<.001$ & 13.8 & 4.5 & $<.001$ & 22.7 & 7.0 & $<.001$ \\
\hline $\begin{array}{l}\text { Neurodegenerative } \\
\text { disease }\end{array}$ & 13.7 & 3.5 & $<.001$ & 12.6 & 2.7 & $<.001$ & 15.9 & 4.7 & $<.001$ & 15.0 & 4.3 & $<.001$ \\
\hline Liver disease & 6.1 & 4.3 & $<.001$ & 5.2 & 3.5 & $<.001$ & 6.6 & 4.6 & $<.001$ & 6.9 & 5.1 & $<.001$ \\
\hline \multicolumn{13}{|l|}{ Health care utilization } \\
\hline Any hospitalization & 25.0 & 8.2 & $<.001$ & 20.5 & 5.1 & $<.001$ & 21.4 & 7.9 & $<.001$ & 28.7 & 10.6 & $<.001$ \\
\hline
\end{tabular}


Table 2 Demographic and clinical characteristics of community-dwelling older patients with chronic pain with or without ADRD stratified by cohort (Continued)

\begin{tabular}{|c|c|c|c|c|c|c|c|c|c|c|c|c|}
\hline \multirow[t]{2}{*}{$\begin{array}{l}\text { Baseline } \\
\text { characteristic }^{a}\end{array}$} & \multicolumn{2}{|c|}{$\begin{array}{l}\text { Patients with } \\
\text { chronic pain, \% }\end{array}$} & \multirow[b]{2}{*}{$\begin{array}{l}P \text { - } \\
\text { value }^{d}\end{array}$} & \multicolumn{2}{|c|}{$\begin{array}{l}\text { Opioid-naive } \\
\text { patients, } \%\end{array}$} & \multirow[b]{2}{*}{$\begin{array}{l}P \text { - } \\
\text { value }^{d}\end{array}$} & \multicolumn{2}{|c|}{$\begin{array}{l}\text { Patients with } \\
\text { neuropathic pain, } \\
\%\end{array}$} & \multirow{3}{*}{$\begin{array}{l}P \text { - } \\
\text { value }^{d}\end{array}$} & \multicolumn{2}{|c|}{$\begin{array}{l}\text { Patients with } \\
\text { opioid } \\
\text { prescription, \% }\end{array}$} & \multirow{3}{*}{$\begin{array}{l}P \text { - } \\
\text { value }^{\mathrm{d}}\end{array}$} \\
\hline & $\begin{array}{l}\text { With } \\
\text { ADRD }\end{array}$ & $\begin{array}{l}\text { Without } \\
\text { ADRD }\end{array}$ & & $\begin{array}{l}\text { With } \\
\text { ADRD }\end{array}$ & $\begin{array}{l}\text { Without } \\
\text { ADRD }\end{array}$ & & $\begin{array}{l}\text { With } \\
\text { ADRD }\end{array}$ & $\begin{array}{l}\text { Without } \\
\text { ADRD }\end{array}$ & & $\begin{array}{l}\text { With } \\
\text { ADRD }\end{array}$ & $\begin{array}{l}\text { Without } \\
\text { ADRD }\end{array}$ & \\
\hline Total no. & 75,258 & 435,870 & & 48,182 & 304,009 & & 9775 & 64,692 & & 27,801 & 197,886 & \\
\hline Any ED visit & 28.3 & 14.0 & $<.001$ & 21.9 & 9.2 & $<.001$ & 26.9 & 14.3 & $<.001$ & 33.5 & 18.1 & $<.001$ \\
\hline $\begin{array}{l}\text { Any hospital surgical } \\
\text { procedure }\end{array}$ & 6.9 & 3.3 & $<.001$ & 4.4 & 1.3 & $<.001$ & 5.3 & 2.6 & $<.001$ & 8.7 & 4.3 & $<.001$ \\
\hline
\end{tabular}

Abbreviations: ADRD Alzheimer disease and related dementias, ED emergency department

${ }^{a}$ Defined as the 6 months prior to the date of a randomly selected chronic pain diagnosis for each patient

Included Hispanic, Asian, Pacific Islander, and Native American individuals

'Measured as primary or secondary diagnosis as the index diagnosis

${ }^{\mathrm{d}}$ Statistical comparisons and $P$-values were calculated using $t$-tests for continuous variables and chi-square tests for categorical variables

$P<.001)$ in patients with vs without ADRD. Among opioid users, the use of long-term opioids, however, varied by residential setting, with higher use in patients with ADRD in communities (41.2\% vs $35.7 \%$; difference, $5.4 \%$ [95\% CI, 4.9 to $6.0 \%] ; P<.001)$ and lower use in those residing in $\mathrm{NHs}(52.5 \%$ vs $58.2 \%$; difference, $-5.7 \%$ [95\% CI, -7.6 to $-3.8 \%] ; P<.001$ ), when compared to their non-ADRD counterparts. A similar pattern was also observed for use of adjuvant analgesics.

\section{Quality measure 1: contraindicated prescription opioids among chronic pain patients}

Among patients with chronic pain, those with ADRD were less likely than their respective non-ADRD counterparts to receive meperidine, propoxyphene, or partial or mixed opioid agonists contraindicated for older adults, in communities $(0.08 \%$ vs $0.12 \%$; difference, $0.04 \%$ [ $95 \% \mathrm{CI},-0.06$ to $-0.01 \%] ; P<.001)$ or $\mathrm{NHs}$ ( $0.05 \%$ vs $0.21 \%$; difference, $-0.2 \%$ [ $95 \% \mathrm{CI},-0.3$ to $0.03 \%$ ] $P<.001$ ), though utilization was generally low (Table 5).

\section{Quality measure 2: strong or high-dose opioids for opioid-naive patients}

Among patients with chronic pain who had no opioids at baseline, those with ADRD were less likely than their respective non-ADRD counterparts to receive longacting or high-dose prescription opioids in communities (1.5\% vs $2.8 \%$; difference, $-1.3 \%$ [95\% CI, -1.4 to $1.1 \%] ; P<.001)$ or $\mathrm{NHs}(2.5 \%$ vs $3.5 \%$; difference, $-1.0 \%$ [95\% CI, -1.7 to $-0.2 \%] ; P=.010)$ (Table 5).

\section{Quality measure 3: long-term opioid use for neuropathic pain}

Among patients who had neuropathic pain as the index diagnosis, those with ADRD were more likely than their non-ADRD counterparts to have long-term use of prescription opioids in communities $(21.7 \%$ vs $19.5 \%$; difference, $2.2 \%$ [95\% CI, 1.3 to $3.1 \%] ; P=.003$ ) (Table 5). By contrast, in NHs, patients with ADRD and neuropathic pain were less likely than their non-ADRD counterparts to have long-term opioid use (26.9\% vs $36.0 \%$; difference, $-9.2 \%$ [95\% CI, -13.6 to $-4.7 \%$; $P<.001$ ) (Table 5). Similar results were observed in sensitivity analyses restricted to individuals with neuropathic pain who had no history of musculoskeletal or idiopathic pain at baseline.

\section{Quality measure 4: concurrent use of opioids and CNS drugs}

Among patients with chronic pain who received prescription opioids, those with ADRD (vs their non-ADRD counterparts) had higher concurrent use of prescription opioids and other CNS-active drugs against guideline recommendations in communities $(44.1 \%$ vs $33.3 \%$; difference, $10.8 \%$ [95\% CI, 10.2 to $11.4 \%$ ]; $P<.001$ ) and NHs $(58.8 \%$ vs $54.1 \%$; difference, $4.7 \%$ [95\% CI, 2.8 to $6.6 \%] ; P<.001$ ) (Table 5). This pattern was similar across different CNS drug classes in communities, with the largest between-group difference in concurrent use of opioids and antidepressants (including selective serotonin reuptake inhibitors [SSRIs] or tricyclic antidepressants [TCAs]) (difference, 7.2\% [95\% CI, 6.7 to 7.7\%]; $P<.001)$, followed by combined use with antipsychotics (difference, 5.8\% [95\% CI, 5.4 to 6.1\%], $P<0.001$ ) and benzodiazepines (difference, $4.2 \%$ [95\% CI, 3.7 to $4.6 \%$; $P<.001)$. In NHs, concurrent opioid and antipsychotic use was significantly higher $(26.2 \%$ vs $11.4 \%$; difference, $14.8 \%$ [95\% CI, 13.5 to $16.1 \%$ ]; $P<.001$ ), but lower combined use of opioids with benzodiazepine $(21.0 \%$ vs 23.2\%; difference, $-2.2 \%$ [95\% CI, -3.8 to $-0.6 \%$; $P<.001)$ or nonbenzodiazepine (6.7\% vs $9.7 \%$; difference, $-3.0 \%$ [95\% CI, -4.1 to $-1.9 \%$ ]; $P<.001$ ) was observed in patients with ADRD versus without. No difference was observed in the combined use of opioids with SSRIs or TCAs between ADRD and non-ADRD groups (39.1\% vs $38.2 \%$; difference, $0.96 \%$ [ $95 \% \mathrm{CI},-0.9$ to $2.8 \%$ ]; $P=$ .307). 


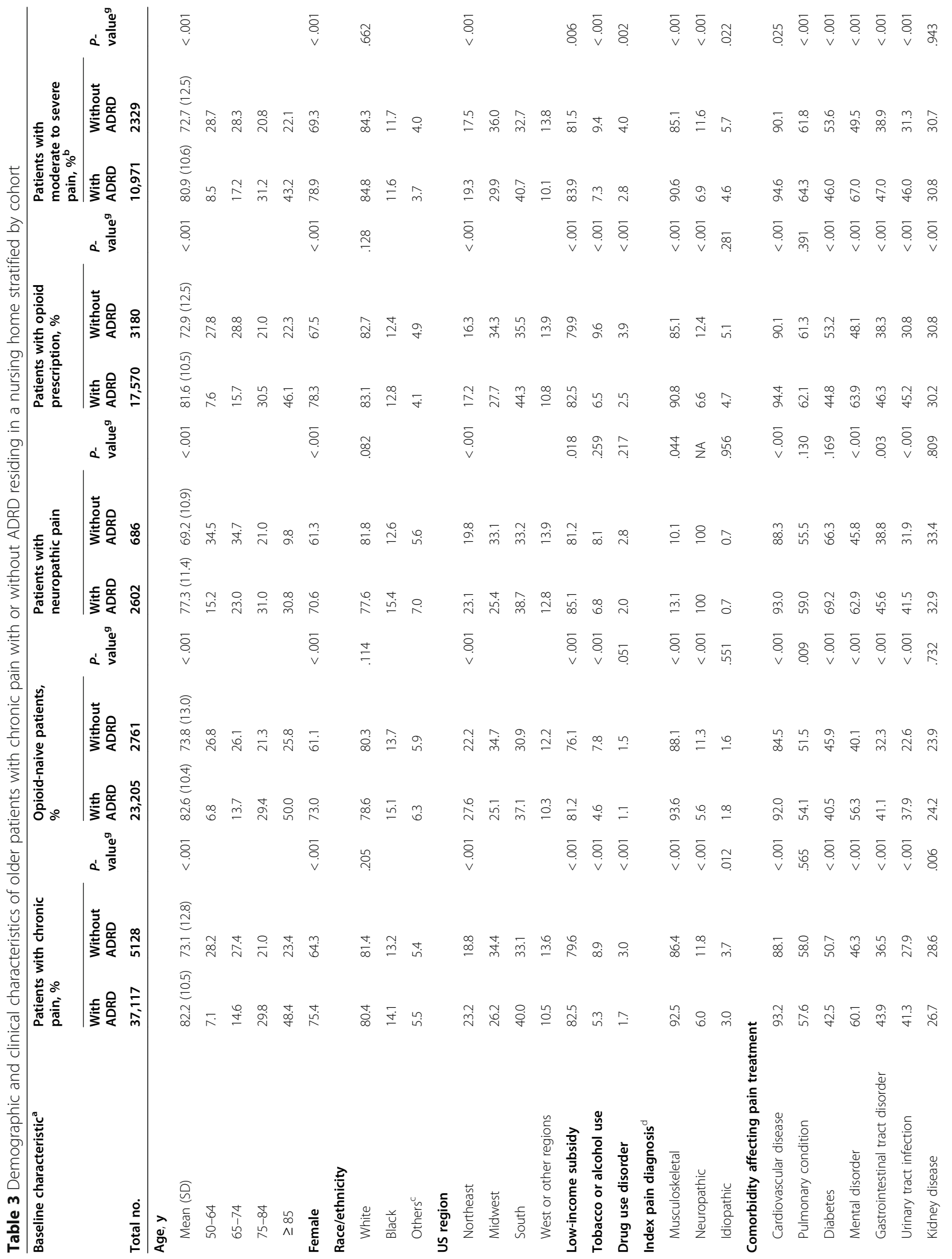




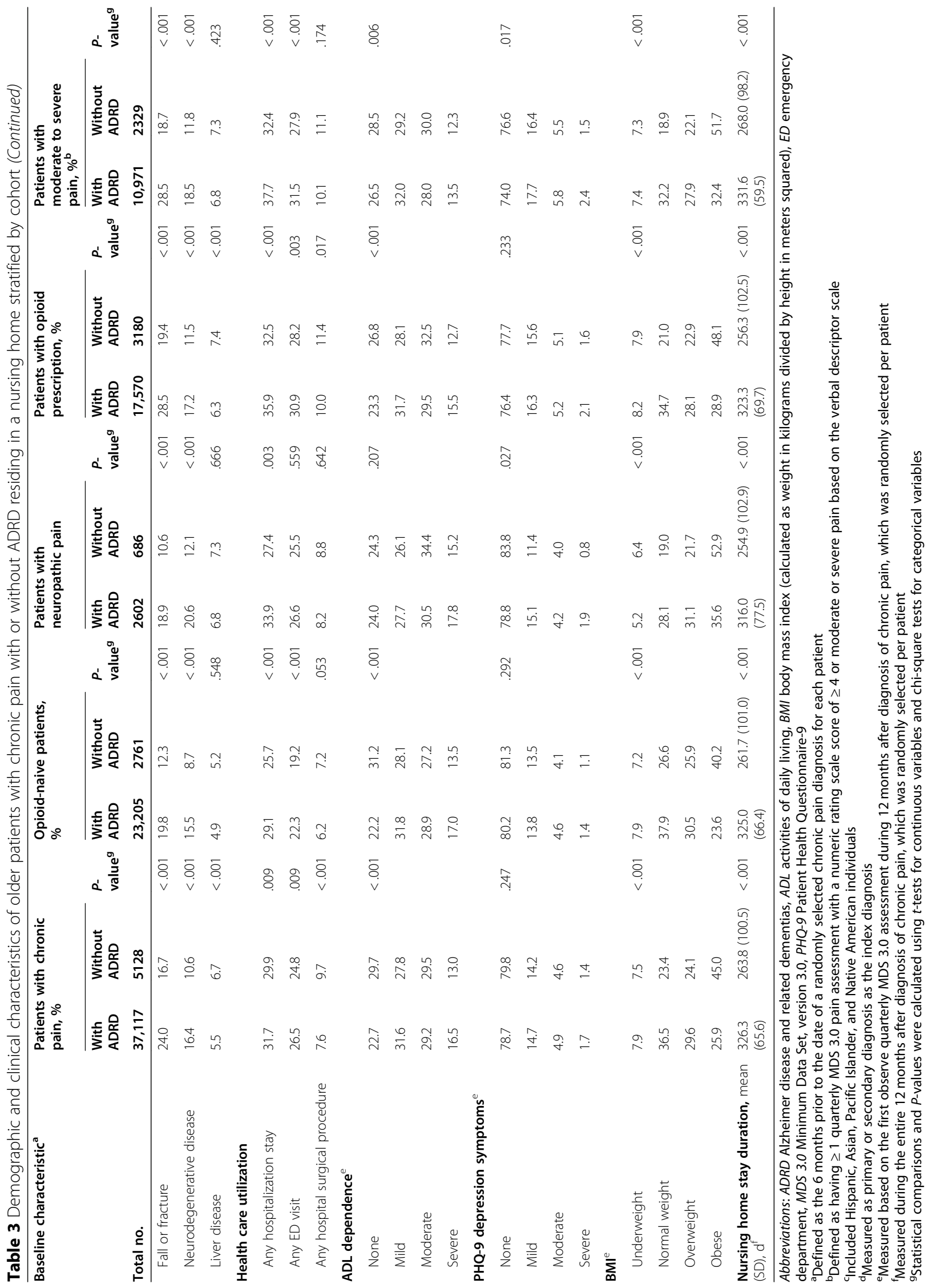


Table 4 Weighted proportion of prescription pain medication use among older patients with chronic pain and with or without ADRD by residential setting

\begin{tabular}{|c|c|c|c|c|c|c|c|c|}
\hline \multirow[t]{2}{*}{ Medication } & \multicolumn{3}{|l|}{ Community } & \multirow[b]{2}{*}{$\begin{array}{l}P \text { - } \\
\text { value }\end{array}$} & \multicolumn{4}{|l|}{ Nursing home } \\
\hline & $\begin{array}{l}\text { Weighted } \\
\text { proportion } \\
\text { among patients } \\
\text { with ADRD, \% }\end{array}$ & $\begin{array}{l}\text { Weighted } \\
\text { proportion among } \\
\text { patients without } \\
\text { ADRD, \% }\end{array}$ & $\begin{array}{l}\text { Proportion } \\
\text { difference } \\
(95 \% \mathrm{Cl})^{\mathrm{a}}\end{array}$ & & $\begin{array}{l}\text { Weighted } \\
\text { proportion } \\
\text { among patients } \\
\text { with ADRD, \% }\end{array}$ & $\begin{array}{l}\text { Weighted } \\
\text { proportion among } \\
\text { patients without } \\
\text { ADRD, \% }\end{array}$ & $\begin{array}{l}\text { Proportion } \\
\text { difference } \\
(95 \% \mathrm{Cl})^{\mathrm{a}}\end{array}$ & $\begin{array}{l}P \text { - } \\
\text { value }\end{array}$ \\
\hline $\begin{array}{l}\text { Any use of } \\
\text { prescription pain } \\
\text { medication }^{b, c}\end{array}$ & 66.6 & 67.6 & $\begin{array}{l}-1.0(-1.4 \\
\text { to }-0.7)\end{array}$ & $<.001$ & 64.5 & 74.9 & $\begin{array}{l}-10.4(- \\
11.7 \text { to }- \\
9.1)\end{array}$ & $<.001$ \\
\hline \multicolumn{9}{|l|}{ Opioid } \\
\hline Any use & 45.1 & 48.1 & $\begin{array}{l}-3.0(-3.4 \\
\text { to }-2.6)\end{array}$ & $<.001$ & 47.9 & 60.7 & $\begin{array}{l}-12.8(- \\
14.2 \text { to }- \\
11.3)\end{array}$ & $<.001$ \\
\hline $\begin{array}{l}\text { Long-term use of } \\
\text { opioid }^{d}\end{array}$ & 41.2 & 35.7 & $\begin{array}{l}5.4(4.9 \text { to } \\
6.0)\end{array}$ & $<.001$ & 52.5 & 58.2 & $\begin{array}{l}-5.7(-7.6 \\
\text { to }-3.8)\end{array}$ & $<.001$ \\
\hline \multicolumn{9}{|l|}{ Non-opioid } \\
\hline Any use & 30.3 & 32.7 & $\begin{array}{l}-2.4(-2.8 \\
\text { to }-2.1)\end{array}$ & $<.001$ & 18.0 & 21.9 & $\begin{array}{l}-3.9(-5.1 \\
\text { to }-2.7)\end{array}$ & $<.001$ \\
\hline \multicolumn{9}{|l|}{ Adjuvant analgesic } \\
\hline Any use & 35.9 & 32.7 & $\begin{array}{l}3.1 \text { ( } 2.8 \text { to } \\
3.5)\end{array}$ & $<.001$ & 33.2 & 40.9 & $\begin{array}{l}-7.6(-9.1 \\
\text { to }-6.2)\end{array}$ & $<.001$ \\
\hline $\begin{array}{l}\text { SNRI or tricyclic } \\
\text { antidepressant }\end{array}$ & 40.0 & 26.8 & $\begin{array}{l}13.1 \text { (12.7 to } \\
13.5)\end{array}$ & $<.001$ & 59.4 & 53.3 & $\begin{array}{l}6.1 \text { (4.6 to } \\
7.6)\end{array}$ & $<.001$ \\
\hline Anticonvulsant & 21.1 & 17.7 & $\begin{array}{l}3.4 \text { (3.1 to } \\
3.7)\end{array}$ & $<.001$ & 21.9 & 27.2 & $\begin{array}{l}-5.3(-6.7 \\
\text { to }-4.0)\end{array}$ & $<.001$ \\
\hline $\begin{array}{l}\text { Skeletal muscle } \\
\text { relaxant }\end{array}$ & 14.2 & 15.6 & $\begin{array}{l}-1.4(-1.7 \\
\text { to }-1.2)\end{array}$ & $<.001$ & 8.2 & 14.8 & $\begin{array}{l}-6.7(-7.7 \\
\text { to }-5.6)\end{array}$ & $<.001$ \\
\hline Topical analgesic $^{\mathrm{e}}$ & 8.1 & 6.6 & $\begin{array}{l}1.5 \text { (1.3 to } \\
1.7)\end{array}$ & $<.001$ & 8.8 & 11.9 & $\begin{array}{l}-3.0(-4.0 \\
\text { to }-2.1)\end{array}$ & $<.001$ \\
\hline
\end{tabular}

Abbreviations: ADRD Alzheimer disease and related dementias, SNRI serotonin-norepinephrine reuptake inhibitor

a Proportion difference between patients with or without ADRD was estimated with generalized linear models with weight statement (to incorporate inverse probability weighting that balances differences in baseline characteristics between the ADRD and non-ADRD groups)

Use of prescription pain medications was measured during the 12 months after diagnosis of chronic pain, which was randomly selected per patient

${ }^{\mathrm{C}} \mathrm{A}$ patient may have more than 1 type of drug combination during the 12-month observation period

${ }^{\mathrm{d}}$ The proportion was calculated among opioid users

ePrescription topical analgesics included diclofenac and lidocaine

\section{Quality measure 5 ( $\mathrm{NH}$ only): opioid prescribing for moderate to severe pain}

Among $\mathrm{NH}$ residents diagnosed as having chronic pain with at least 1 episode of moderate to severe pain during the year after a chronic pain diagnosis, those with ADRD were less likely than those without ADRD to have opioids prescribed during the 30 days before or after reporting moderate to severe pain, or to have a scheduled pain medication regimen in 5 days before the pain episode (60.1\% vs $52.5 \%$; difference, $7.6 \%$ [95\% CI, 5.3-9.9\%]; $P<.001)$ (Table 5).

\section{Discussion}

In this nationally representative study of older Medicare patients with chronic noncancer pain between 2011 and 2015, we found differences in adherence to current pain guidelines between patients with and without ADRD, but the magnitude and direction of the differences varied across the indicators of potentially inappropriate opioid prescribing. Notably, of the five indicators, two measures-concurrent use of prescription opioids and other CNS-active drugs and no scheduled opioids for moderate or severe pain-were more common among patients with ADRD than among patients without ADRD in community or NH settings. The other three measures of potentially inappropriate prescribing were similar or lower in patients with ADRD than in those without in either setting.

The concurrent use of prescription opioids and CNSactive drugs was prevalent (between 33 and 59\%) in our study population residing in the community or $\mathrm{NH}$ setting. The estimated prevalence of concurrent opioidCNS drug use echoes recent studies, suggesting a rise in CNS polypharmacy that involved opioids in older adults and in patients with dementia [33, 34]. A potential reason for our observed higher concurrent opioid-CNS drug use in ADRD may be its non-cognitive neuropsychiatric symptoms (NPS) (e.g., agitation, psychosis, 
Table 5 Weighted proportion of inappropriate opioid prescribing practice for patients with or without ADRD by residential setting

\begin{tabular}{|c|c|c|c|c|c|c|c|c|}
\hline \multirow{2}{*}{$\begin{array}{l}\text { Quality indicators of } \\
\text { inappropriate opioid } \\
\text { prescribing }^{\mathrm{a}}\end{array}$} & \multicolumn{3}{|l|}{ Community } & \multirow{2}{*}{$\begin{array}{l}P \text { - } \\
\text { value }\end{array}$} & \multicolumn{3}{|l|}{ Nursing home } & \multirow{2}{*}{$\begin{array}{l}P \text { - } \\
\text { value }\end{array}$} \\
\hline & $\begin{array}{l}\text { Weighted } \\
\text { proportion } \\
\text { among patients } \\
\text { with ADRD, } \%\end{array}$ & $\begin{array}{l}\text { Weighted } \\
\text { proportion } \\
\text { among patients } \\
\text { without ADRD, \% }\end{array}$ & $\begin{array}{l}\text { Proportion } \\
\text { difference } \\
(95 \% \mathrm{Cl})^{\mathrm{b}}\end{array}$ & & $\begin{array}{l}\text { Weighted } \\
\text { proportion } \\
\text { among patients } \\
\text { with ADRD, \% }\end{array}$ & $\begin{array}{l}\text { Weighted } \\
\text { proportion } \\
\text { among patients } \\
\text { without ADRD, \% }\end{array}$ & $\begin{array}{l}\text { Proportion } \\
\text { difference } \\
(95 \% \mathrm{CI})^{\mathrm{b}}\end{array}$ & \\
\hline
\end{tabular}

Use of opioids contraindicated for older adults with chronic pain

\begin{tabular}{|c|c|c|c|c|c|c|c|}
\hline $\begin{array}{l}\text { Use of meperidine, } \\
\text { propoxyphene or } \\
\text { partial or mixed } \\
\text { opioid agonsists }\end{array}$ & 0.08 & 0.12 & $\begin{array}{l}-0.04(- \\
0.06 \text { to }- \\
0.01)\end{array}$ & $<.001$ & 0.05 & 0.21 & $\begin{array}{l}-0.2(-0.3 \\
\text { to }-0.03)\end{array}$ \\
\hline
\end{tabular}

\section{Opioid prescribing for opioid-naive patients}

\begin{tabular}{|c|c|c|c|c|c|c|c|c|}
\hline $\begin{array}{l}\text { Use of long-acting } \\
\text { opioid }\end{array}$ & 0.49 & 0.62 & $\begin{array}{l}-0.13(- \\
0.20 \text { to }- \\
0.06)\end{array}$ & $<.001$ & 1.3 & 1.8 & $\begin{array}{l}-0.5(-1.0 \\
\text { to } 0.04)\end{array}$ & .070 \\
\hline $\begin{array}{l}\text { Use of high-dose } \\
\text { opioid }\end{array}$ & 1.3 & 2.5 & $\begin{array}{l}-1.2(-1.3 \\
\text { to }-1.1)\end{array}$ & $<.001$ & 1.9 & 2.6 & $\begin{array}{l}-0.7(-1.3 \\
\text { to }-0.05)\end{array}$ & .035 \\
\hline Composite of either & 1.5 & 2.8 & $\begin{array}{l}-1.3(-1.4 \\
\text { to }-1.1)\end{array}$ & $<.001$ & 2.5 & 3.5 & $\begin{array}{l}-1.0(-1.7 \\
\text { to }-0.2)\end{array}$ & .010 \\
\hline
\end{tabular}

\section{Opioid prescribing for patients with neuropathic pain as the index diagnosis}

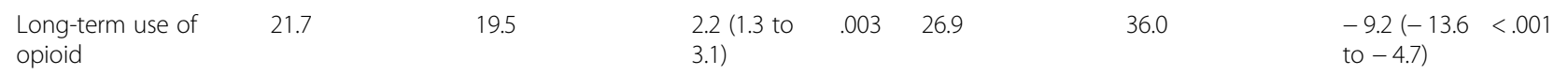

Sensitivity analysis: for patients with neuropathic pain without baseline musculoskeletal or idiopathic pain

\begin{tabular}{|c|c|c|c|c|c|c|}
\hline $\begin{array}{l}\text { Long-term use of } \\
\text { opioid }\end{array}$ & 12.1 & 10.4 & $\begin{array}{l}1.8 \text { ( } 0.6 \text { to } \\
2.9)\end{array}$ & $<.001 \quad 25.7$ & 33.1 & $\begin{array}{l}-7.5(- \\
12.1 \text { to }- \\
2.8)\end{array}$ \\
\hline
\end{tabular}

Concurrent use of opioid and CNS depressant for opioid users

\begin{tabular}{|c|c|c|c|c|c|c|c|c|}
\hline $\begin{array}{l}\text { With any qualifying } \\
\text { CNS depressant }^{c}\end{array}$ & 44.1 & 33.3 & $\begin{array}{l}10.8(10.2 \text { to } \\
11.4)\end{array}$ & $<.001$ & 58.8 & 54.1 & $\begin{array}{l}4.7(2.8 \text { to } \\
6.6)\end{array}$ & $<.001$ \\
\hline With benzodiazepine & 19.5 & 15.3 & $\begin{array}{l}4.2(3.7 \text { to } \\
4.6)\end{array}$ & $<.001$ & 21.0 & 23.2 & $\begin{array}{l}-2.2(-3.8 \\
\text { to }-0.6)\end{array}$ & $<.001$ \\
\hline With SSRI or TCA & 26.8 & 19.6 & $\begin{array}{l}7.2(6.7 \text { to } \\
7.7)\end{array}$ & $<.001$ & 39.1 & 38.2 & $\begin{array}{l}0.96(-0.9 \\
\text { to } 2.8)\end{array}$ & .307 \\
\hline With antipsychotic & 10.4 & 4.6 & $\begin{array}{l}5.8(5.4 \text { to } \\
6.1)\end{array}$ & $<.001$ & 26.2 & 11.4 & $\begin{array}{l}14.8(13.5 \text { to } \\
16.1)\end{array}$ & $<.001$ \\
\hline $\begin{array}{l}\text { With } \\
\text { nonbenzodiazepine }\end{array}$ & 9.5 & 8.2 & $\begin{array}{l}1.3 \text { (1.0 to } \\
1.7)\end{array}$ & $<.001$ & 6.7 & 9.7 & $\begin{array}{l}-3.0(-4.1 \\
\text { to }-1.9)\end{array}$ & $<.001$ \\
\hline \multicolumn{9}{|c|}{ Opioid or other scheduled analgesic regimen for moderate to severe pain ${ }^{d}$} \\
\hline $\begin{array}{l}\text { No use of prescription } \\
\text { opioid }^{e}\end{array}$ & ND & ND & ND & ND & 32.7 & 24.6 & $\begin{array}{l}8.1(6.1 \text { to } \\
10.1)\end{array}$ & $<.001$ \\
\hline $\begin{array}{l}\text { No use of scheduled } \\
\text { pain medication }{ }^{f}\end{array}$ & ND & ND & ND & ND & 47.8 & 43.1 & $\begin{array}{l}4.7(2.4 \text { to } \\
7.0)\end{array}$ & $<.001$ \\
\hline Composite of either & ND & ND & ND & ND & 60.1 & 52.5 & $\begin{array}{l}7.6(5.3 \text { to } \\
9.9)\end{array}$ & $<.001$ \\
\hline
\end{tabular}

Abbreviations: ADRD Alzheimer disease and related dementias, CNS central nervous system, MDS 3.0 Minimum Data Set, version 3.0, MME morphine milligram equivalent, ND not determined, SNRI serotonin-norepinephrine reuptake inhibitor, SSRI selective serotonin reuptake inhibitor, TCA tricyclic antidepressant ${ }^{a}$ Measured during the 12 months after diagnosis of chronic pain randomly selected per patient

bProportion difference between ADRD and non-ADRD groups was estimated with generalized linear models along with weight statement (to incorporate inverse probability weighting that balances differences in baseline characteristics between the ADRD and non-ADRD groups)

'CNS-active drugs included antipsychotics, benzodiazepine, nonbenzodiazepine or hypnotics, tricyclic antidepressants, and SNRIs

${ }^{\mathrm{d}}$ Defined as having at least 1 quarterly MDS 3.0 pain assessment with a numeric rating scale score of 4 or more, or moderate or severe pain based on a verbal descriptor scale

${ }^{\mathrm{e}}$ Measured as having at least 1 quarterly MDS 3.0 moderate to severe pain score without prescription opioids dispensed within 30 days before and after the MDS 3.0 pain assessment

${ }^{\mathrm{f}}$ Measured as having at least 1 quarterly MDS 3.0 moderate to severe pain score that had no scheduled pain medications (assessed in MDS 3.0 Section J)

depression, anxiety, and sleep disturbance) [35], and thus a greater perceived need for psychotropic medications [36]. While the use of psychotropics in ADRD is controversial due to concerns over safety associated with these drugs [37], antipsychotics are indicated for aggression and psychosis and antidepressants are for major 
depression and anxiety. Literature has documented the safety of concurrent use of opioids and benzodiazepines in older populations with or without ADRD [38-40]. However, to our best knowledge, no population-based studies have examined benefits and harms associated with concurrent use of opioids and antipsychotics or antidepressants, the most common drug combination in patients with ADRD observed by the present study. Given the high prevalence of ADRD patients who had comorbid chronic pain and NPS symptoms [2], future research is needed to identify risk factors and effects of potentially inappropriate concurrent opioid-psychotropic use on patient outcomes.

Among NH residents who reported moderate or severe pain, we observed that over half had no opioid prescribing nor scheduled pain medication regimen within several days of the pain reporting, with a higher proportion seen in patients with ADRD than those without. Our finding echoes previous studies, underscoring the long-standing concern on potential undertreatment of pain in patients with ADRD [7, 41]. It seems that proactive treatment with scheduled pain medications, including opioids, was less common in patients with ADRD than those without. The underlying causes for this disparity are unclear and may be related to health providers' knowledge of and attitudes toward prescribing opioids for patients with ADRD [42-44]. Recent literature suggests over half of primary care doctors were uncertain about the safety of using opioids to treat pain in dementia patients, and many disagreed with prescribing analgesics regularly, even if this approach is considered the optimal treatment of pain [42]. Our results highlight that many $\mathrm{NH}$ residents with moderate or severe pain, particularly those with ADRD, might be at high risk of having their chronic pain undertreated.

Our study observed one in four community-dwelling older adults with neuropathic pain receiving long-term opioids in patients with or without ADRD. Our estimate is consistent with literature indicating that $22.7 \%$ of patients with dementia and polyneuropathy receive longterm opioid therapy [45]. Clinical guidelines often list opioids as a later-line treatment for neuropathic pain after failure of adjuvant and non-opioid therapy [46]. No guideline has endorsed long-term opioid use owing to limited evidence of efficacy, opioid dependency, and overdose concern [45]. Notably, we observed an opposite pattern in $\mathrm{NHs}$, with lower use of long-term opioids among patients with than those without ADRD. The observed findings may be explained by differences in clinician specialties, with general or family medicine physicians being more likely to provide treatment for patients in communities, whereas geriatricians or advanced practitioners (nurse practitioners and physician assistants) being more likely to provide treatment in NHs [47].

The present study also compared the prevalence of receiving any prescription pain medications during the year after a chronic pain diagnosis and found a small difference between community-dwelling patients with and without ADRD (66.6\% vs 67.6\%). While the difference was statistically significant largely due to our large sample size, the magnitude of the difference was too small to indicate any clinical significance. Our estimate in the community population is consistent with that of a recent population-based study, suggesting that patients with ADRD were as likely to receive pain treatment as patients without ADRD in the community [7]. In NHs, we also observed $64.5 \%$ of residents with ADRD receiving pain treatment, although the figure was lower than that (74.9\%) of residents without ADRD. The discrepancy in the use of pain treatment between $\mathrm{NH}$ residents with and without ADRD may be explained by differences in pain severity, cognitive function, and communication ability $[9,48]$. The loss of verbal communication skills likely occurs among patients in the late stages of ADRD, leading to great difficulties in detecting pain [12]. Our estimate of pain medication use in the $\mathrm{NH}$ population is more aligned with data of recent studies [49] than those of earlier research [50].

\section{Implications}

Our study has important implications for clinical and research purposes. Clinically, the use of prescription pain therapy, including opioids, among patients with ADRD has increased from 30-56\% between 2006 and 2010 [41, 50,51 to $67-90 \%$ between 2011 and 2016, observed in this and a recent study [49]. Such increase may reflect great improvements in awareness of pain assessment and management for older adults with ADRD, which have been emphasized for years by governments [52] and professional societies [24-26, 52]. Regarding opioid prescribing quality, clinical recommendations for avoiding strong or high-dose opioids for opioid-naïve patients and contraindicated opioids for older adults appear to translate well into clinical practice, with only $4 \%$ of older adults with or without ADRD receiving such inappropriate prescribing practices. Contrary to what the guidelines suggest, many patients with ADRD received opioids concurrently with other CNS drugs or received no scheduled opioids for moderate or severe pain. These discrepancies could be explained by many reasons, including time lag in adapting, lack of awareness, and disagreement on guidelines [53], which rely on evidence largely from cognitively intact older adults [12]. From research perspectives, it remains unclear whether these deviated opioid prescribing practices are associated with outcomes of patients with ADRD. Perhaps, the 
fundamental question that ought to be answered is whether opioids are safe, especially if used concurrently with psychotropic CNS drugs for neuropsychiatric symptoms (NPSs), which affects 95\% of patients with ADRD [35]. These questions need to be addressed with considerations of limited treatment options available for NPSs, shortened life expectancy [54], and health outcomes (e.g., pain control, physical independence) that are attainable and desirable in patients with ADRD [55].

\section{Strengths and limitations}

The Medicare claims data lacked information on some important elements, such as prior pain management experience (e.g., response and tolerance to opioids) and medical notes (e.g., drug and disease contraindications to opioids) that may justify deviations from guidelines. Although lacking these data may have hindered our ability to assess the quality of opioid prescribing, we mitigated this issue by balancing characteristics derived from Medicare claims or MDS 3.0 data, thus achieving comparison groups with presumably similar distributions of these factors that may explain deviations from guidelines. Analyses in a specific residential setting also helped reduce the heterogeneity of patient characteristics when comparing quality measures between patients with ADRD or without. Second, baseline depression and functional ability measured using MDS 3.0 were accounted for in the $\mathrm{NH}$ sample but not the community sample due to lack of information on these variables. Third, Medicare data do not detail the indication for which a drug was prescribed, which creates difficulty in determining the type of pain condition (e.g., neuropathic or nociceptive pain) for which the opioids were prescribed among patients with multiple co-existing pain conditions. Fourth, while MDS 3.0 used validated tools (i.e., numerical rating scale or verbal descriptor scale) to capture self-reported pain from $\mathrm{NH}$ patients who are capable of communication, these tools may not completely capture pain severity from patients with ADRD, particularly those in later stages where memory and communication ability is deteriorating [12]. Fifth, our results are derived from Medicare fee-for-service beneficiaries and cannot be generalized to those with Medicare Advantage or the non-Medicare population. Sixth, the assessed quality indicators of opioid prescribing may act against each other. For example, not prescribing opioids for patients with moderate to severe pain violates one guideline recommendation, but such practice may be necessary to avoid opioid use with other existing CNS drugs. Finally, the study used data prior to 2016, and it is unclear to what extent the 2016 Centers for Disease Control and Prevention guideline has shaped the quality of opioid prescribing in patients with ADRD and chronic pain. Studies exploring the impact of this more recent federal guidance are needed.

\section{Conclusions}

Potential inappropriate opioid prescribing in 2 of 5 areas of pain care was more common among patients with ADRD than among patients without ADRD in community or NH settings between 2011 and 2015. Further studies exploring determinants and health outcomes associated with opioid prescribing in identified areas of pain care among patients with ADRD are warranted.

\section{Supplementary Information}

Supplementary information accompanies this paper at https://doi.org/10. 1186/s13195-021-00818-3.

Additional file 1: Table S1. ICD-9-CM Codes and Procedures for Disease Conditions and Service Care Considered in the Study. Table S2. Medications of Interest in This Study. Table S3. Current and Recent Guideline and Consensus Documents on Opioid Prescribing for Older Adults with Noncancer pain. Table S4. Characteristics of CommunityDwelling Patients with Chronic Pain With or Without ADRD Using Inverse Propensity Weighting, by Cohort. Table S5. Characteristics of Nursing Home Residents Who Have Chronic Pain With or Without ADRD Using Inverse Propensity Weighting, by Cohort. Figure S1. Flowchart of Included Patients. Figure S2. Absolute Standardized Differences for Baseline Demographic and Clinical Characteristics of Community-Dwelling Patients With Chronic Pain With or Without ADRD in the Original Population and After Inverse Probability Weighting. Figure S3. Absolute Standardized Differences for Baseline Demographic and Clinical Characteristics of Nursing Home Residents Who Had Chronic Pain With or Without ADRD in the Original Population and After Inverse Probability Weighting.

\section{Acknowledgements}

None.

\section{Sponsor's role}

The National Institute on Aging had no role in the design and conduct of the study; collection, management, analysis, and interpretation of the data; preparation, review, or approval of the manuscript; or the decision to submit the manuscript for publication.

\section{Authors' contributions}

Study concept and design (Wei and Winterstein), acquisition of subjects and/ or data (Wei), data analysis (Wei and Chen), interpretation of data (all authors), and preparation of the manuscript (all authors). The authors read and approved the final manuscript.

\section{Funding}

This project was funded by a Mentored Research Scientist Award (K01AG054764, Dr. Wei) from the National Institute on Aging.

\section{Availability of data and materials}

The data that support the findings of this study are available from the Centers for Medicare and Medicaid Services but restrictions apply to the availability of these data, which were used under license for the current study, and so are not publicly available.

\section{Declarations}

Ethics approval and consent to participate

The University of Florida Institutional Review Board approved and waived patient informed consent for this study.

Consent for publication

Not applicable. 


\section{Competing interests}

The authors declare that they have no competing interests.

\section{Author details}

'Department of Pharmaceutical Outcomes and Policy, University of Florida College of Pharmacy, 1225 Center Drive, Health Professions Nursing Pharmacy Building, Room 3321, Gainesville, FL 32610, USA. ${ }^{2}$ Center for Drug Evaluation and Safety, University of Florida, Gainesville, FL, USA. ${ }^{3}$ Department of Community Health and Family Medicine, College of Medicine, University of Florida, Gainesville, FL, USA. ${ }^{4}$ Pain Research and Intervention Center of Excellence, University of Florida, Gainesville, USA. ${ }^{5}$ College of Dentistry, University of Florida, Gainesville, FL, USA. ${ }^{6}$ Division of Geriatrics and Palliative Medicine, Weill Cornell Medical College, New York, NY, USA. 'Department of Neurology, McKnight Brain Institute, University of Florida, Gainesville, FL, USA. ${ }^{8} \mathrm{NF} / \mathrm{SG}$ Veterans Health System, Malcom Randall VAMC, Geriatrics Research, Education, Clinical Center (GRECC), Gainesville, FL, USA. ${ }^{9}$ University of Florida College of Nursing, Gainesville, FL, USA. ${ }^{10}$ Department of Aging and Geriatric Research, Institute on Aging, University of Florida College of Medicine, Gainesville, FL, USA. ${ }^{11}$ Department of Biostatistics, University of Florida Colleges of Medicine and Public Health \& Health Professions, Gainesville, FL, USA. ${ }^{12}$ Department of Epidemiology, University of Florida Colleges of Medicine and Public Health \& Health Professions, Gainesville, FL, USA.

Received: 8 August 2020 Accepted: 26 March 2021

Published online: 12 April 2021

\section{References}

1. Takai Y, Yamamoto-Mitani N, Okamoto Y, Koyama K, Honda A. Literature review of pain prevalence among older residents of nursing homes. Pain Manag Nurs. 2010;11(4):209-23. https://doi.org/10.1016/j.pmn.2010.08.006.

2. van Dalen-Kok AH, Pieper MJ, de Waal MW, Lukas A, Husebo BS, Achterberg WP. Association between pain, neuropsychiatric symptoms, and physical function in dementia: a systematic review and meta-analysis. BMC Geriatr. 2015;15(1):49. https://doi.org/10.1186/s12877-015-0048-6.

3. van Kooten J, van der Wouden JC, Sikkes SAM, Smalbrugge M, Hertogh C, Stek ML. Pain, neuropsychiatric symptoms, and quality of life of nursing home residents with advanced dementia in the Netherlands: a crosssectional study. Alzheimer Dis Assoc Disord. 2017;31(4):315-21. https://doi. org/10.1097/WAD.0000000000000197.

4. Reid MC, Eccleston C, Pillemer K. Management of chronic pain in older adults. BMJ. 2015;350(2):h532. https://doi.org/10.1136/bmj.h532.

5. Tan EC, Jokanovic N, Koponen MP, Thomas D, Hilmer SN, Bell JS. Prevalence of analgesic use and pain in people with and without dementia or cognitive impairment in aged care facilities: a systematic review and metaanalysis. Curr Clin Pharmacol. 2015;10(3):194-203. https://doi.org/10.2174/1 57488471003150820144958.

6. Scherder E, Oosterman J, Swaab D, Herr K, Ooms M, Ribbe M, Sergeant J, Pickering G, Benedetti F. Recent developments in pain in dementia. BMJ. 2005;330(7489):461-4. https://doi.org/10.1136/bmj.330.7489.461.

7. Haasum Y, Fastbom J, Fratiglioni L, Kareholt I, Johnell K. Pain treatment in elderly persons with and without dementia: a population-based study of institutionalized and home-dwelling elderly. Drugs Aging. 2011;28(4):283-93. https://doi.org/10.2165/11587040-000000000-00000.

8. Griffioen C, Husebo BS, Flo E, Caljouw MAA, Achterberg WP. Opioid prescription use in nursing home residents with advanced dementia. Pain Med. 2019;20(1):50-7. https://doi.org/10.1093/pm/pnx268.

9. Ersek M, Nash PV, Hilgeman MM, Neradilek MB, Herr KA, Block PR, Collins AN. Pain patterns and treatment among nursing home residents with moderate-severe cognitive impairment. J Am Geriatr Soc. 2020;68(4):794802. https://doi.org/10.1111/jgs.16293.

10. Jensen-Dahm C, Gasse C, Astrup A, Mortensen PB, Waldemar G. Frequent use of opioids in patients with dementia and nursing home residents: a study of the entire elderly population of Denmark. Alzheimers Dement. 2015;11(6):691-9. https://doi.org/10.1016/j.jalz.2014.06.013.

11. Barry HE, Parsons C, Passmore AP, Hughes CM. Exploring the prevalence of and factors associated with pain: a cross-sectional study of communitydwelling people with dementia. Health Soc Care Community. 2016;24(3): 270-82. https://doi.org/10.1111/hsc.12204.

12. Achterberg W, Lautenbacher $S$, Husebo B, Erdal A, Herr K. Pain in dementia. Pain Rep. 2020;5(1):e803. https://doi.org/10.1097/PR9.0000000000000803.
13. Moriya AS, Miller GE. Any use and frequent use of opioids among elderly adults in 2015-2016, by socioeconomic characteristics. Rockville: Statistical Brief (Medical Expenditure Panel Survey (US)); 2018.

14. The Centers for Medicare and Medicaid Services. Chronic Condition Data Warehouse Chronic Categories 2020 [cited 2020. Available from: https:// www2.ccwdata.org/web/guest/condition-categories.

15. Rahman AN, Applebaum RA. The nursing home Minimum Data Set assessment instrument: manifest functions and unintended consequences-past, present, and future. Gerontologist. 2009;49(6):727-35. https://doi.org/1 0.1093/geront/gnp066.

16. Gruber-Baldini AL, Stuart B, Zuckerman $1 H$, Simoni-Wastila L, Miller R. Treatment of dementia in community-dwelling and institutionalized medicare beneficiaries. J Am Geriatr Soc. 2007;55(10):1508-16. https://doi. org/10.1111/j.1532-5415.2007.01387.x.

17. Olsen C, Pedersen I, Bergland A, Enders-Slegers MJ, Joranson N, Calogiuri G, et al. Differences in quality of life in home-dwelling persons and nursing home residents with dementia - a cross-sectional study. BMC Geriatr. 2016; 16(1):137. https://doi.org/10.1186/s12877-016-0312-4.

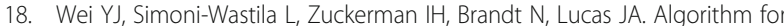
identifying nursing home days using Medicare claims and minimum data set assessment data. Med Care. 2016;54(11):e73-e7. https://doi.org/10.1097/ MLR.0000000000000109.

19. The Centers for Medicare and Medicaid Senvices. Opioid oral morphine milligram equivalent (MME) conversion factors 2020. Available from: https//wuw.cms.gov/ Medicare/Prescription-Drug-Coverage/PrescriptionDrugCovContra/Downloads/OralMME-CFs-vFeb-2018.pdf. Accessed 1 Jan 2020.

20. Dowell D, Haegerich TM, Chou R. CDC guideline for prescribing opioids for chronic pain--United States, 2016. JAMA. 2016;315(15):1624-45. https://doi. org/10.1001/jama.2016.1464.

21. Braden JB, Russo J, Fan MY, Edlund MJ, Martin BC, DeVries A, Sullivan MD. Emergency department visits among recipients of chronic opioid therapy. Arch Intern Med. 2010;170(16):1425-32. https://doi.org/10.1001/a rchinternmed.2010.273.

22. Sullivan MD, Edlund MJ, Fan MY, Devries A, Brennan Braden J, Martin BC. Risks for possible and probable opioid misuse among recipients of chronic opioid therapy in commercial and medicaid insurance plans: the TROUP study. Pain. 2010;150(2):332-9. https://doi.org/10.1016/j.pain.2010.05.020.

23. Gellad WF, Zhao X, Thorpe CT, Thorpe JM, Sileanu FE, Cashy JP, Mor M, Hale JA, Radomski T, Hausmann LRM, Fine MJ, Good CB. Overlapping buprenorphine, opioid, and benzodiazepine prescriptions among veterans dually enrolled in Department of Veterans Affairs and Medicare Part D. Subst Abus. 2017;38(1):22-5. https://doi.org/10.1080/08897077.2016.1267071.

24. American Geriatrics Society Beers Criteria Update Expert P. American Geriatrics Society 2015 updated Beers criteria for potentially inappropriate medication use in older adults. J Am Geriatr Soc. 2015;63(11):2227-46. https://doi.org/10.1111/jgs.13702.

25. American Geriatrics Society Beers Criteria Update Expert P. American Geriatrics Society updated Beers criteria for potentially inappropriate medication use in older adults. J Am Geriatr Soc. 2012;60(4):616-31. https:// doi.org/10.1111/j.1532-5415.2012.03923.x.

26. American Geriatrics Society Panel on Pharmacological Management of Persistent Pain in Older P. Pharmacological management of persistent pain in older persons. J Am Geriatr Soc. 2009;57(8):1331-46.

27. Chou R. 2009 clinical guidelines from the American Pain Society and the American Academy of Pain Medicine on the use of chronic opioid therapy in chronic noncancer pain: what are the key messages for clinical practice? Pol Arch Med Wewn. 2009;119(7-8):469-77.

28. Curtis LH, Hammill BG, Eisenstein EL, Kramer JM, Anstrom KJ. Using inverse probability-weighted estimators in comparative effectiveness analyses with observational databases. Med Care. 2007;45(10 Supl 2):S103-7.

29. Kroenke K, Spitzer RL, Williams JB. The PHQ-9: validity of a brief depression severity measure. J Gen Intern Med. 2001;16(9):606-13. https://doi.org/10.1 046/j.1525-1497.2001.016009606.x.

30. Carpenter Gl, Hastie CL, Morris JN, Fries BE, Ankri J. Measuring change in activities of daily living in nursing home residents with moderate to severe cognitive impairment. BMC Geriatr. 2006;6(1):7. https:/doi.org/10.1186/1471-2318-6-7.

31. Obesity: preventing and managing the global epidemic. Report of a WHO consultation. World Health Organ Tech Rep Ser. 2000;894:i-xii, 1-253.

32. Petersen ML, Porter KE, Gruber S, Wang Y, van der Laan MJ. Diagnosing and responding to violations in the positivity assumption. Stat Methods Med Res. 2012;21(1):31-54. https://doi.org/10.1177/0962280210386207. 
33. Gerlach LB, Olfson M, Kales HC, Maust DT. Opioids and other central nervous system-active polypharmacy in older adults in the United States. J Am Geriatr Soc. 2017;65(9):2052-6. https://doi.org/10.1111/jgs.14930.

34. Maust DT, Gerlach LB, Gibson A, Kales HC, Blow FC, Olfson M. Trends in central nervous system-active polypharmacy among older adults seen in outpatient care in the United States. JAMA Intern Med. 2017;177(4):583-5. https://doi.org/10.1001/jamainternmed.2016.9225.

35. Steinberg M, Corcoran C, Tschanz JT, Huber C, Welsh-Bohmer K, Norton MC, Zandi P, Breitner JCS, Steffens DC, Lyketsos CG. Risk factors for neuropsychiatric symptoms in dementia: the Cache County Study. Int J Geriatr Psychiatry. 2006;21(9):824-30. https://doi.org/10.1002/gps.1567.

36. Maust DT, Langa KM, Blow FC, Kales HC. Psychotropic use and associated neuropsychiatric symptoms among patients with dementia in the USA. Int J Geriatr Psychiatry. 2017;32(2):164-74. https://doi.org/10.1002/gps.4452.

37. Masopust J, Protopopova D, Valis M, Pavelek Z, Klimova B. Treatment of behavioral and psychological symptoms of dementias with psychopharmaceuticals: a review. Neuropsychiatr Dis Treat. 2018;14:1211-20. https://doi.org/10.2147/NDT.S163842.

38. Karttunen N, Taipale H, Hamina A, Tanskanen A, Tiihonen J, Tolppanen AM, Hartikainen S. Concomitant use of benzodiazepines and opioids in community-dwelling older people with or without Alzheimer's disease-a nationwide register-based study in Finland. Int J Geriatr Psychiatry. 2019; 34(2):280-8. https://doi.org/10.1002/gps.5018.

39. Rhee TG. Coprescribing of benzodiazepines and opioids in older adults: rates, correlates, and national trends. J Gerontol A Biol Sci Med Sci. 2019: 74(12):1910-5. https://doi.org/10.1093/gerona/gly283.

40. Maree RD, Marcum ZA, Saghafi E, Weiner DK, Karp JF. A systematic review of opioid and benzodiazepine misuse in older adults. Am J Geriatr Psychiatry. 2016;24(11):949-63. https://doi.org/10.1016/j.jagp.2016.06.003.

41. Shen C, Zhao X, Dwibedi N, Wiener RC, Findley PA, Sambamoorthi U. Opioid use and the presence of Alzheimer's disease and related dementias among elderly Medicare beneficiaries diagnosed with chronic pain conditions. Alzheimers Dement (N Y). 2018;4:661-8.

42. Jennings AA, Linehan M, Foley $T$. The knowledge and attitudes of general practitioners to the assessment and management of pain in people with dementia. BMC Fam Pract. 2018;19(1):166. https://doi.org/10.1186/s12875-01 8-0853-z.

43. Burns M, Mcllfatrick S. Nurses' knowledge and attitudes towards pain assessment for people with dementia in a nursing home setting. Int J Palliat Nurs. 2015;21(10):479-87. https://doi.org/10.12968/ijpn.2015.21.10.479.

44. Barry HE, Parsons C, Passmore AP, Hughes CM. Community pharmacists and people with dementia: a cross-sectional survey exploring experiences, attitudes, and knowledge of pain and its management. Int J Geriatr Psychiatry. 2013;28(10):1077-85. https://doi.org/10.1002/gps.3931.

45. Hoffman EM, Watson JC, St Sauver J, Staff NP, Klein CJ. Association of longterm opioid therapy with functional status, adverse outcomes, and mortality among patients with polyneuropathy. JAMA Neurol. 2017;74(7):773-9. https://doi.org/10.1001/jamaneurol.2017.0486.

46. Bates D, Schultheis BC, Hanes MC, Jolly SM, Chakravarthy KV, Deer TR, Levy RM, Hunter CW. A comprehensive algorithm for management of neuropathic pain. Pain Med. 2019;20(Suppl 1):S2-S12. https://doi.org/10.1 093/pm/pnz075.

47. Ryskina KL, Polsky D, Werner RM. Physicians and advanced practitioners specializing in nursing home care, 2012-2015. JAMA. 2017;318(20):2040-2. https://doi.org/10.1001/jama.2017.13378.

48. Bauer U, Pitzer S, Schreier MM, Osterbrink J, Alzner R, Iglseder B. Pain treatment for nursing home residents differs according to cognitive state a cross-sectional study. BMC Geriatr. 2016;16(1):124. https://doi.org/10.1186/ s12877-016-0295-1.

49. Hunnicutt JN, Ulbricht CM, Tjia J, Lapane KL. Pain and pharmacologic pain management in long-stay nursing home residents. Pain. 2017;158(6):1091-9. https://doi.org/10.1097/j.pain.0000000000000887.

50. Monroe TB, Misra SK, Habermann RC, Dietrich MS, Cowan RL, Simmons SF. Pain reports and pain medication treatment in nursing home residents with and without dementia. Geriatr Gerontol Int. 2014;14(3):541-8. https://doi. org/10.1111/ggi.12130.

51. Reynolds KS, Hanson LC, DeVellis RF, Henderson M, Steinhauser KE. Disparities in pain management between cognitively intact and cognitively impaired nursing home residents. J Pain Symptom Manag. 2008;35(4):38896. https://doi.org/10.1016/j.jpainsymman.2008.01.001.
52. Harris $Y$, Clauser SB. Achieving improvement through nursing home quality measurement. Health Care Financ Rev. 2002;23(4):5-18.

53. Cabana MD, Rand CS, Powe NR, Wu AW, Wilson MH, Abboud PA, et al. Why don't physicians follow clinical practice guidelines? A framework for improvement. JAMA. 1999;282(15):1458-65. https://doi.org/10.1001/jama.2 82.15.1458.

54. Rait G, Walters K, Bottomley C, Petersen I, lliffe S, Nazareth I. Survival of people with clinical diagnosis of dementia in primary care: cohort study. BMJ. 2010;341(aug05 2):c3584. https://doi.org/10.1136/bmj.c3584.

55. Jennings LA, Palimaru A, Corona MG, Cagigas XE, Ramirez KD, Zhao T, Hays RD, Wenger NS, Reuben DB. Patient and caregiver goals for dementia care. Qual Life Res. 2017;26(3):685-93. https://doi.org/10.1007/s11136-016-1471-7.

\section{Publisher's Note}

Springer Nature remains neutral with regard to jurisdictional claims in published maps and institutional affiliations.
Ready to submit your research? Choose BMC and benefit from:

- fast, convenient online submission

- thorough peer review by experienced researchers in your field

- rapid publication on acceptance

- support for research data, including large and complex data types

- gold Open Access which fosters wider collaboration and increased citations

- maximum visibility for your research: over $100 \mathrm{M}$ website views per year

At BMC, research is always in progress.

Learn more biomedcentral.com/submissions 